\title{
MOF Compound RiMO-301
}

National Cancer Institute

\section{Source}

National Cancer Institute. MOF Compound RiMO-301. NCI Thesaurus. Code C148280.

A nanoparticle-based metal-organic framework (MOF) compound composed of proprietary X-ray-absorbing metals, with potential radiosensitizing properties. Upon intratumoral administration and subsequent irradiation of the tumor site, RiMO-301 absorbs the X-ray photons and produces reactive oxygen species (ROS), such as hydroxyl radicals and sing let oxygen, which induces ROS-mediated DNA damage in the irradiated cancer cells leading to tumor cell lysis. In addition, RiMO-301 may also contain an as of yet unidentified immunomodulating agent loaded into the channels/pores of the construct that may induce an immune response against the tumor-associated antigens (TAAs) released by the lysed tumor cells, thereby locally killing additional tumor and nontumor cells. MOFs, porous crystalline materials composed of metal clusters and organic linkers, generate ROS at much lower X-ray dosages than used in standard radiotherapy, which results in reduced radiation exposure and X-ray damage to normal, healthy cells. 